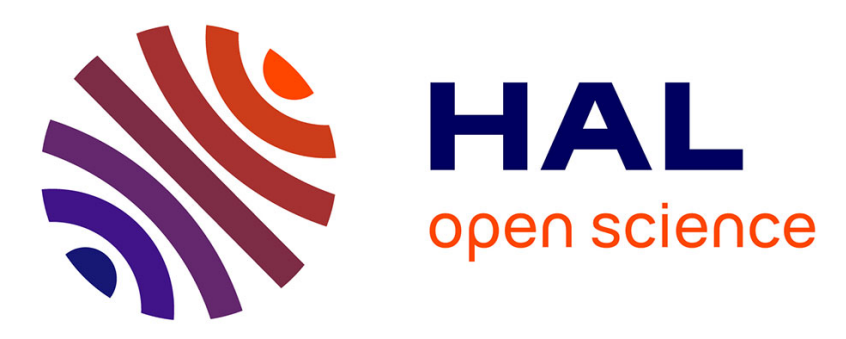

\title{
From Relations Between Sets to Relations Between Belief Functions
}

\author{
Sébastien Destercke, Frédéric Pichon, John Klein
}

\section{To cite this version:}

Sébastien Destercke, Frédéric Pichon, John Klein. From Relations Between Sets to Relations Between Belief Functions. 5th International Conference on Belief Functions (BELIEF 2018), Sep 2018, Compiègne, France. pp.65-72, 10.1007/978-3-319-99383-6_9 . hal-02053069

\section{HAL Id: hal-02053069 \\ https://hal.science/hal-02053069}

Submitted on 21 Jun 2021

HAL is a multi-disciplinary open access archive for the deposit and dissemination of scientific research documents, whether they are published or not. The documents may come from teaching and research institutions in France or abroad, or from public or private research centers.
L'archive ouverte pluridisciplinaire HAL, est destinée au dépôt et à la diffusion de documents scientifiques de niveau recherche, publiés ou non, émanant des établissements d'enseignement et de recherche français ou étrangers, des laboratoires publics ou privés. 


\title{
From relations between sets to relations between belief functions
}

\author{
Sébastien Destercke ${ }^{1}$, Frédéric Pichon ${ }^{2}$, and John Klein ${ }^{3}$ \\ 1 Technologic University of Compiègne, CNRS, UMR 7253 - Heudiasyc, Centre de \\ Recherche de Royallieu, Compiègne, France \\ 2 Univ. Artois, EA 3926, Laboratoire de Génie Informatique et d'Automatique de \\ l'Artois (LGI2A), Béthune, F-62400, France. \\ 3 Univ. Lille, CNRS, Centrale Lille, UMR 9189 - CRIStAL - Centre de Recherche en \\ Informatique Signal et Automatique de Lille, F-59000 Lille, France \\ sebastien.destercke@hds.utc.fr,frederic.pichon@univ-artois.fr, \\ john.klein@univ-lille1.fr
}

\begin{abstract}
In uncertainty theories, a common problem is to define how we can extend relations between sets (e.g., inclusion, ranking, consistency, ... ) to corresponding notions between uncertainty representations. Such definitions can then be used to perform the same operations as those that are done for sets: measuring information content, ordering alternatives or checking consistency, to name a few. In this paper, we propose a general way to extend set relations to belief functions, using constrained stochastic matrices to identify those belief functions in relation. We then study some properties of our proposal, as well as its relations with existing works focusing on peculiar relations.
\end{abstract}

Keywords: set relations, belief functions, specificity, ranking, consistency.

\section{Introduction}

One can define many relations between two (or more) subsets $A, B$ of some finite set $X$, i.e. between elements of some boolean algebra $\left(2^{X}, \cap, \cup, . C\right)$. Such relations can check whether the sets are consistent $(A \cap B \neq \emptyset)$; whether one set is more informative than another, or imply one another $(A \subseteq B)$; when the space on which they are defined is ordered, whether one set is "higher" than another $(A \prec B)$; etc. These relations can then be related to practical problems such as restoring consistency or ranking alternatives.

To address the same questions in those uncertainty theories that formally generalize set theory (based, e.g., on possibility distributions, belief functions or sets of probabilities), it is desirable to carry over relations between sets to uncertainty representations. Given the higher expressiveness of such theories, the problem is ill-posed in the sense that there is not a unique way to do so. We can cite as a typical example the notion of inclusion between belief functions, that has many definitions [3]. Yet, such works usually focus on extending one particular relation in meaningful ways. 
In this paper, we propose a simple way to extend any set relation to an equivalent relation between belief functions, in the sense that the relation is exactly recovered when considering categorical belief functions (i.e., belief functions reduced to one focal element). Basically, we require that for a pair of belief functions to be in relation, there must exist at least one stochastic matrix such that one of these belief functions is obtained as the dot product of the matrix with the other belief function. Additionally, the matrix is constrained to have non-null entries on pairs of focal sets satisfying the relation to extend.

We develop and study the properties of our proposal in Section 2, in which we also include necessary reminders. We then show in Section 3 how this proposal is linked to previously proposed relations between belief functions, as well as to other related results. We will focus, in particular, on the notions of information specificity, of consistency, and of ranking. Finally, we formalize in Section 4 how we can say whether a relation is preserved through functional mapping of a variable to another one, and provide some results about the inclusion and ranking cases.

\section{Main proposal}

\subsection{Definitions}

A belief function on a finite space $X=\left\{x_{1}, \ldots, x_{K}\right\}$ is in one-to-one correspondence with a mass function $m_{i}: 2^{X} \rightarrow[0,1]$ that satisfies $\sum_{A \subseteq X} m_{i}(A)=1$. From such a mass function, the belief and plausibility of an event $A$ respectively read

$$
\operatorname{Bel}_{i}(A)=\sum_{E \subseteq A} m_{i}(E) \text { and } P l_{i}(A)=\sum_{E \cap A \neq \emptyset} m_{i}(E) .
$$

If $m_{i}(\emptyset)=0$, they can be interpreted as bounds of the probability $P(A)$ of $A$, inducing the probability set $\mathcal{P}_{i}=\left\{P: \operatorname{Bel}_{i}(A) \leq P(A) \leq P l_{i}(A), \forall A \subseteq X\right\}$. We denote by $\mathcal{B}^{X}$ the set of all belief functions on $X$. A particularly interesting subclass of belief functions for this study will be the one of categorical ones. A categorical mass function, denoted $m_{B}$, is such that $m_{B}(B)=1$.

Let us now consider a relation such that for any ordered pair $(A, B) \subseteq X^{2}$, we will denote by $A \mathbf{R} B$ the truth of the relation between $A$ and $B$ ( $\mathbf{R}$ is thus a binary relation on $2^{X}$, or equivalently a subset of $\left.2^{X} \times 2^{X}\right)$. We then propose the following simple definition to extend this relation to belief functions, i.e. into a relation on $\mathcal{B}^{X}$ :

Definition 1 Given two mass functions $m_{1}, m_{2}$ and a subset relation $\mathbf{R}$, we say that $m_{1} \tilde{\mathbf{R}} m_{2}$ iff there is a (left) ${ }^{4}$ stochastic matrix $S_{\mathbf{R}}$ such that $\forall A, B \subseteq X$

$$
\begin{aligned}
& m_{1}(A)=\sum_{B \subseteq X} S_{\mathbf{R}}(A, B) m_{2}(B) \\
& \text { with } S(A, B)>0 \wedge m_{2}(B)>0 \Longrightarrow A \mathbf{R} B .
\end{aligned}
$$

\footnotetext{
${ }^{4}$ We use left-stochasticity only throughout the paper.
} 
It is easily checked that $\tilde{\mathbf{R}}$ is a generalisation of $\mathbf{R}$ in the sense that

$$
m_{A} \tilde{\mathbf{R}} m_{B} \Leftrightarrow A \mathbf{R} B, \forall A, B \subseteq X .
$$

Indeed, if $A \mathbf{R} B$, we can choose $S_{\mathbf{R}}(E, F)=m_{A}(E)$ and this matrix matches the conditions of Definition 1 , hence $m_{A} \tilde{\mathbf{R}} m_{B}$. Also, there is only one relation $\tilde{\mathbf{R}}$ on belief functions spanned by Definition 1 from a given set relation $\mathbf{R}$. Suppose two such belief function relations exist. If a matrix matching the conditions of Definition 1 was found for the first one then the same matrix also works for the other and the relations are equivalent. Similarly, if $\tilde{\mathbf{R}}$ is spanned by Definition 1 from a given set relation $\mathbf{R}$ then it cannot be spanned by other set relations in the same way. This is an immediate consequence of (3). Consequently, we will use the same notation for a relation $\mathbf{R}$ on the subset or belief function side in the remainder of the paper.

Definition 1 is inspired from previous works on specificity of belief functions $[3,4,6]$, as well as on recent proposals dealing with belief function ordering [5]. As these works dealt with directional, or rather asymmetric relations, Definition 1 is naturally asymmetric. However, Proposition 1 shows that it has a somehow symmetric counterpart.

Proposition 1. Consider two mass functions $m_{1}, m_{2}$ and a belief function relation $\mathbf{R}$. Then the two following conditions are equivalent:

1. there is a stochastic matrix $S_{\mathbf{R}}(A, B)$ such that

$$
\begin{gathered}
m_{1}(A)=\sum_{B \subseteq X} S_{\mathbf{R}}(A, B) m_{2}(B), \\
\text { with } S_{\mathbf{R}}(A, B)>0 \wedge m_{2}(B)>0 \Longrightarrow A \mathbf{R} B .
\end{gathered}
$$

2. there is a joint mass function $m_{12}$ on $2^{X} \times 2^{X}$ such that

$$
\begin{gathered}
m_{12}(A, B)>0 \Longrightarrow A \mathbf{R} B, \\
m_{1}(A)=\sum_{B} m_{12}(A, B), \\
m_{2}(B)=\sum_{A} m_{12}(A, B) .
\end{gathered}
$$

Proof (Sketch). 1. $\Longrightarrow 2$ : from a matrix $S_{\mathbf{R}}(A, B)$, we can deduce a joint $m_{12}(A, B)=m_{2}(B) S_{\mathbf{R}}(A, B)$ for any $A, B$ which satisfies 2 .

2 . $\Longrightarrow 1$ : from a joint $m_{12}(A, B)$ satisfying (4)-(6), define $S_{\mathbf{R}}(A, B)=$ $m_{12}(A, B) / m_{2}(B)$ if $m_{2}(B)>0$, and with arbitrary values making it (left)-stochastic if $m_{2}(B)=0$. This matrix satisfies 1 .

This proposition shows, in particular, that any stochastic matrix $S_{\mathbf{R}}$ can be associated to a unique joint mass function $m_{12}$, and vice-versa. Also note that, using a transformation similar to the one used in the proof, we can build a stochastic matrix $S_{\mathbf{R}}^{\prime}$ such that $S_{\mathbf{R}}^{\prime}(B, A)=m_{12}(A, B) / m_{1}(A)$ if $m_{1}(A)>0$, and be with arbitrary values else. $S^{\prime}$ is such that $m_{2}(B)=\sum_{A \subseteq X} S_{\mathbf{R}}^{\prime}(B, A) m_{1}(A)$ with $S_{\mathbf{R}}^{\prime}(A, B)>0$ and $m_{1}(A)>0$ implying $B \mathbf{R} A$, but not necessarily $A \mathbf{R} B$. 


\subsection{Relation properties preservation}

We may now wonder how much of the initial relation $\mathbf{R}$ properties between sets do still exist when extended in this way to belief functions. We will now provide a series of results for common properties, either by providing proofs or counter-examples. We will keep the proposition/proof format, to provide a uniform presentation.

Proposition 2 (Preserved symmetry). If $\mathbf{R}$ is symmetric on sets, it is so on belief functions:

$$
m_{1} \mathbf{R} m_{2} \equiv m_{2} \mathbf{R} m_{1}, \forall m_{1}, m_{2}
$$

Proof (Sketch). If $\mathbf{R}$ is symmetric, then $S_{\mathbf{R}}^{\prime}(A, B)$ as defined above is such that $S_{\mathbf{R}}^{\prime}(A, B)>0$ and $m_{1}(A)>0$ implies $A \mathbf{R} B$.

Proposition 3 (Unpreserved antisymmetry). If $\mathbf{R}$ is antisymmetric on sets, it is not necessarily so on belief functions, as we may have

$$
m_{1} \mathbf{R} m_{2} \wedge m_{2} \mathbf{R} m_{1} \text { and } m_{2} \neq m_{1}
$$

Proof. Consider two mass functions that are positives only on subsets $A, B, C$ and such that

$$
\begin{aligned}
& m_{1}(A)=0.3, m_{1}(B)=0.5, m_{1}(C)=0.2, \\
& m_{2}(A)=0.4, m_{2}(B)=0.3, m_{2}(C)=0.3,
\end{aligned}
$$

and the antisymmetric relation $\mathbf{R}$ on $A, B, C$ summarised by the matrix

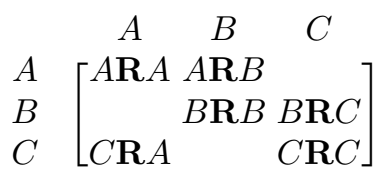

We can then build two different joint mass functions such that $m_{1} \mathbf{R} m_{2}$ and $m_{2} \mathbf{R} m_{1}$.

Proposition 4 (unpreserved asymmetry). If $\mathbf{R}$ is asymmetric on sets, it is not necessarily so on belief functions, as we may have

$$
m_{1} \mathbf{R} m_{2} \text { and } m_{2} \mathbf{R} m_{1}
$$

Proof. Simply consider two mass functions $m_{1}, m_{2}$ that are positive only on subsets $A, B, C, D, E$ and such that

$$
\begin{aligned}
& m_{1}(A)=0.2, m_{1}(B)=0.3, m_{1}(C)=0.2, m_{1}(D)=0.1, m_{1}(E)=0.2, \\
& m_{2}(A)=0.2, m_{2}(B)=0.1, m_{2}(C)=0.3, m_{2}(D)=0.3, m_{2}(E)=0.1
\end{aligned}
$$


as well as the asymmetric relation $\mathbf{R}$ on those subsets summarised by the matrix

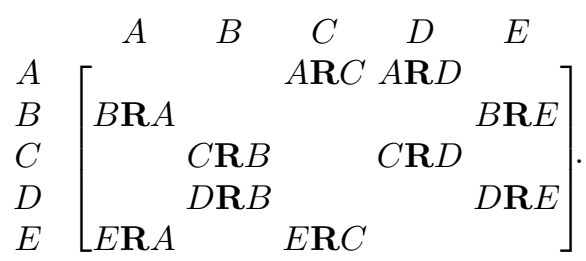

We can then build two different joint mass functions such that $m_{1} \mathbf{R} m_{2}$ and $m_{2} \mathbf{R} m_{1}$.

Proposition 5 (Preserved reflexivity). If $\mathbf{R}$ is reflexive on sets, it is so on belief functions:

$$
\forall m \text {, we have } m \mathbf{R} m
$$

Proof (sketch). Just consider the joint mass function $m_{12}(A, A)=m(A)$ if $m_{1}=$ $m_{2}=m$.

Proposition 6 (Unpreserved irreflexivity). If $\mathbf{R}$ is irreflexive on sets, it is not necessarily so on belief functions, as we may have $m \mathbf{R} m$ for some $m \in \mathcal{B}$.

Proof. Consider the following mass function

$$
m\left(A_{1}\right)=0.5, m\left(A_{2}\right)=0.5
$$

and the relation $\mathbf{R}$ summarised in the following matrix

$$
\begin{aligned}
& A_{1} \\
& A_{2}
\end{aligned}\left[\begin{array}{cc}
A_{1} & A_{2} \\
A_{2} \mathbf{R} A_{1} & A_{1} \mathbf{R} A_{2}
\end{array}\right]
$$

which is irreflexive. However, the joint $m\left(A_{1}, A_{2}\right)=m\left(A_{2}, A_{1}\right)=0.5$ shows that we have $m \mathbf{R} m$, hence $\mathbf{R}$ may not be irreflexive for belief functions.

Proposition 7 (Preserved transitivity). If $\mathbf{R}$ is transitive on sets, it is so on belief functions:

$$
m_{1} \mathbf{R} m_{2} \wedge m_{2} \mathbf{R} m_{3} \Longrightarrow m_{1} \mathbf{R} m_{3}
$$

Proof (sketch). Consider two stochastic matrices $S_{\mathbf{R}_{12}}$ and $S_{\mathbf{R}_{23}}$ satisfying Definition 1, we can show that their product gives a matrix $S_{\mathbf{R}_{13}}$ satisfying Definition 1.

Proposition 8 (Unpreserved completeness). If $\mathbf{R}$ is complete (or total) on sets, it is not necessarily so on belief functions: for any two $m_{1}, m_{2}$ we may have neither $m_{1} \mathbf{R} m_{2}$ nor $m_{2} \mathbf{R} m_{1}$. 
Proof. Consider the following mass functions

$$
m_{1}\left(A_{1}\right)=0.6, m_{1}\left(A_{2}\right)=0.4 ; m_{2}\left(B_{1}\right)=m_{2}\left(B_{2}\right)=0.5
$$

and the relation $\mathbf{R}$ summarised in the following matrix

$$
\begin{aligned}
& B_{1} \quad B_{2} \\
& \begin{array}{l}
A_{1} \\
A_{2}
\end{array} \quad\left[\begin{array}{ll}
A_{1} \mathbf{R} B_{1} & B_{2} \mathbf{R} A_{1} \\
B_{1} \mathbf{R} A_{2} & A_{2} \mathbf{R} B_{2}
\end{array}\right]
\end{aligned}
$$

It is clear that any joint mass function respecting conditions (5)-(6) must give a non-null mass to both $\left(A_{1}, B_{1}\right)$ and $\left(A_{1}, B_{2}\right)$, hence we have neither $m_{1} \mathbf{R} m_{2}$ nor $m_{2} \mathbf{R} m_{1}$.

Table 1 summarises our obtained results. Note that some properties unpreserved in general can nevertheless be preserved in peculiar cases (e.g., antisymmetry of inclusion is preserved, as specialisation is antisymmetric). This opens the way to various further questions (i.e., what happens when considering poset structures).

\begin{tabular}{cc||cc}
$\mathbf{R}$ on $2^{X}$ is & $\rightarrow \mathbf{R}$ on $\mathcal{B}^{X}$ is & $\mathbf{R}$ on $2^{X}$ is $\rightarrow \mathbf{R}$ on $\mathcal{B}^{X}$ is \\
\hline Symmetric & Yes & Irreflexive & No \\
Antisymmetric & No & Transitive & Yes \\
Asymmetric & No & Complete & No \\
Reflexive & Yes & & \\
\hline
\end{tabular}

\section{Related works}

\subsection{Inclusion and consistency}

In the case where the relations are either inclusion or consistency, then we retrieve well-known results of the literature:

- in the case of inclusion we have $A \mathbf{R} B$ iff $A \subseteq B$, our definition is essentially the same as specialisation [3], since checking $m_{2}(B)>0$ is unnecessary in this case.

- in the case of consistency, we have $A \mathbf{R} B$ iff $A \cap B \neq \emptyset$, and one can see that $m_{1} \mathbf{R} m_{2}$ iff there is a joint mass affecting positive mass to pairs of sets having a non-empty intersection. This is equivalent to require $\mathcal{P}_{1} \cap \mathcal{P}_{2} \neq \emptyset$, with $\mathcal{P}_{i}$ the probability set induced by $m_{i}[1]$. 


\section{$3.2 \quad$ Rankings}

When the space $X$ is ordered (with $x_{i} \leq x_{i+1}$ ) and possibly infinite, it makes sense to consider relations of the kind "higher than" in order to compare sets.

There are many ways to rank two sets $A, B$, such as:

- Single-bound dominance, that can be declined itself into four notions:

- loose dominance: $A \mathbf{R}_{\leq_{L D}} B$ if $\min A \leq \max B$

- lower bound: $A \mathbf{R}_{\leq_{L B}} B$ if $\min A \leq \min B$

- upper bound: $A \mathbf{R}_{\leq_{U P}} B$ if $\max A \leq \max B$

- strict dominance: $A \mathbf{R}_{\leq_{S D}} B$ if $\max A \leq \min B$

- Pairwise-bound or lattice dominance: $A \mathbf{R}_{\leq_{P D}} B$ if $\min A \leq \min B$ and $\max A \leq$ $\max B$, whose extension to belief functions studied in [5] correspond to our proposal.

Extensions of this kind are connected to the extensions of stochastic dominance explored in [2].

\section{Preservation by functional mapping}

In this section, we investigate how we can check whether a relation is preserved by a functional mapping, in the univariate case (multivariate case easily follows). Such mappings are indeed used in lots of applications involving uncertainty propagation (e.g., multi-criteria decision making, risk analysis, ...).

Let $f$ be some function with domain $X$ and codomain $Y$, i.e., $f: X \rightarrow Y$. The image $f(A)$ of $A \subseteq X$ under $f$ is the subset $f(A)=\{f(x): x \in A\} \subseteq Y$. More generally, the image $f(m)$ of some mass function $m \in \mathcal{B}^{X}$ under $f$ is the mass function $f(m) \in \mathcal{B}^{Y}$ defined as

$$
f(m)(B)=\sum_{f(A)=B} m(A) \text { for all } B \subseteq Y .
$$

Definition 1. Let $f: X \rightarrow Y$. Let $\mathbf{R}^{X}$ and $\mathbf{R}^{Y}$ be relations on $2^{X}$ and $2^{Y}$, respectively. The pair $\left(\mathbf{R}^{X}, \mathbf{R}^{Y}\right)$ of relations $\mathbf{R}^{X}$ and $\mathbf{R}^{Y}$ is said to be compatible with respect to $f$ ( $f$-compatible for short) if, for all $A, B \subseteq X$,

$$
A \mathbf{R}^{X} B \Rightarrow f(A) \mathbf{R}^{Y} f(B) .
$$

Example 1. Let $\mathbf{R}_{\subseteq}^{X}$ be the relation corresponding to inclusion on $X$, i.e., $A \mathbf{R}_{\subseteq}^{X} B$ iff $A \subseteq B, A, B \subseteq X$. Similarly, let $\mathbf{R}_{\subseteq}^{Y}$ denote inclusion on $Y$. Since for any function $f$ and any $A, B \subseteq X$ such that $A \subseteq B$ it holds that $f(A) \subseteq f(B)$, the pair $\left(\mathbf{R}_{\subseteq}^{X}, \mathbf{R}_{\subseteq}^{Y}\right)$ is $f$-compatible for any $f$. Similarly, the pair $\left(\mathbf{R}_{\subset}^{X}, \mathbf{R}_{\subseteq}^{Y}\right)$ is $f$-compatible for any $f$.

Now, let $X$ and $Y$ be two ordered spaces and let $\mathbf{R}_{\leq{ }_{P D}}^{X}$ and $\mathbf{R}_{\leq P D}^{Y}$ be the relations corresponding to pairwise-bound dominance on $X$ and on $Y$, respectively. Then, the $f$-compatibility of pair $\left(\mathbf{R}_{\leq_{P D}}^{X}, \mathbf{R}_{\leq_{P D}}^{Y}\right)$ depends on $f$. In particular, if $f$ is monotonically non-decreasing, we have $f(A) \leq_{P D} f(B)$ for all $A, B \subseteq X$ 
such that $A \leq_{P D} B$, and thus the pair $\left(\mathbf{R}_{\leq_{P D}}^{X}, \mathbf{R}_{\leq_{P D}}^{Y}\right)$ is $f$-compatible for such $f$. However, this pair is not $f$-compatible when $f$ is monotonically non-increasing since in general we have in this case $A \leq_{P D} B \nRightarrow f(A) \leq_{P D} f(B)$.

Proposition 9 (Preserved compatibility). If $\left(\mathbf{R}^{X}, \mathbf{R}^{Y}\right)$ is $f$-compatible, it is so on belief functions:

$$
m_{1} \mathbf{R}^{X} m_{2} \Rightarrow f\left(m_{1}\right) \mathbf{R}^{Y} f\left(m_{2}\right) .
$$

Proof (sketch). Consider a joint mass $m_{12}$ on $X$ satisfying satisfying (4)-(6) for $\mathbf{R}^{X}$. Then if $\mathbf{R}^{X}, \mathbf{R}^{Y}$ is $f$-compatible, mapping $m_{12}$ through $f$ results in a joint mass showing that $f\left(m_{1}\right) \mathbf{R}^{Y} f\left(m_{2}\right)$.

We note that Proposition 9 and its straightforward extension to multivariate functions (not presented here due to lack of space) generalize results in $[4,5]$ concerning inclusion and ranking.

\section{Conclusion}

In this paper, a very general way to extend a binary relation on sets to a binary relation on belief functions is introduced. Several results are provided to assess which properties of the relation are preserved through this mechanism. Our proposal is also connected to more specific generalisation of binary relations, such as the notion of specialisation. Consequently, our results are also a generalisation of pre-existing ones for specific relations.

We believe that the original ideas presented in this paper shall reach out a large audience of belief function practitioners wishing to address multi-criteria decision making, reliability analysis or optimisation problems, in which some relations such as ranking or information loss relations can play a significant role.

\section{References}

1. Chateauneuf, A.: Combination of compatible belief functions and relation of specificity. In: Advances in the Dempster-Shafer theory of evidence. pp. 97-114. John Wiley \& Sons, Inc. (1994)

2. Denœux, T.: Extending stochastic ordering to belief functions on the real line. Information Sciences 179(9), 1362-1376 (2009)

3. Dubois, D., Prade., H.: A set-theoretic view of belief functions: logical operations and approximations by fuzzy sets. International Journal of General Systems 12(3), 193-226 (1986)

4. Dubois, D., Prade, H.: Random sets and fuzzy interval analysis. Fuzzy Sets and Systems 42, 87-101 (1991)

5. Helal, N., Pichon, F., Porumbel, D., Mercier, D., Lefèvre., E.: The capacitated vehicle routing problem with evidential demands. International Journal of Approximate Reasoning 95, 124-151 (2018)

6. Yager, R.R.: The entailment principle for Dempster-Shafer granules. International Journal of Intelligent Systems 1(4), 247-262 (1986) 